\title{
Neurointervention in the Era of COVID-19: Korean Nationwide Survey, Literature Review, and Recommendations
}

\author{
Woong Jae Lee, $\mathrm{MD}, \mathrm{PhD}^{1}$, Jun Soo Byun, $\mathrm{MD}, \mathrm{PhD}^{2}$ \\ 1 Department of Radiology, H Plus YangJi Hospital, Seoul, Korea \\ ${ }^{2}$ Department of Radiology, Chung-Ang University Hospital, Chung-Ang University College of Medicine, Seoul, Korea
}

\begin{abstract}
The coronavirus disease 2019 (COVID-19) pandemic caused by severe acute respiratory syndrome coronavirus 2 (SARS-CoV-2) has had a tremendous impact on healthcare systems worldwide. Although the most common presentation of COVID-19 is respiratory illness, neurologic manifestations are increasing and the pandemic may have consequential effects on urgent conditions such as acute ischemic stroke. In this document, we describe the current status of neurointervention in Korea affected by COVID-19 based on a nationwide survey and review relevant literature from other countries and professional societies.
\end{abstract}

Key Words: COVID-19; Surveys and questionnaires; Review; Stroke; Therapeutics

\section{INTRODUCTION}

Currently, Korea is in the era of its 4th pandemic with the coronavirus disease 2019 (COVID-19). As of August 6, 2021, the cumulative number of confirmed patients in Korea is 207,406, with more than 1,500 confirmed cases per day, resulting in 2,113 deaths. However, according to the Healthcare Bigdata Hub of Health Insurance Review and Assessment Service (HIRA), there was a mild increase in the number of aneurysm coilings in 2019 and 2020 (14,384 in 2019 and 15,348 in 2020) and mechanical thrombectomy for acute stroke $(4,954$ in 2019 and 5,420 in 2020).

Screening for fever and respiratory symptoms should be part of the screening of all potential neurointervention patients. The reverse-transcription polymerase chain reaction testing (RTP(R) with a nasal and pharyngeal swab is the current diagnostic gold standard for confirming infection with COVID-19. However, since this test takes several hours, many stroke patients tend to receive a rapid antigen test and chest computed tomography (CT) for emergency neurointervention, which can give results within minutes to tens of minutes.

Patients with suspected or confirmed COVID-19 must always wear a mask. It is recommended to use a dedicated route (including elevators) from the emergency department to the angiography suite for patients with suspected or confirmed COVID-19.' The physical and mental stress of healthcare workers during the COVID-19 pandemic is a substantial problem. A recent study to

\author{
Correspondence to: \\ Jun Soo Byun, MD, PhD \\ Department of Radiology, Chung-Ang \\ University Hospital, 102 Heukseok-ro, \\ Dongjak-gu, Seoul 06973, Korea \\ Tel: +82-2-6299-2643 \\ Fax: +82-2-6263-1557 \\ Email: flightdr61@cau.ac.kr \\ Received: June 16, 2021 \\ Revised: August 6, 2021 \\ Accepted: August 11, 2021 \\ Copyright $\odot 2021$ Korean Society of \\ Interventional Neuroradiology \\ This is an Open Access article distributed under the terms of \\ the Creative Commons Attribution Non-Commercial License \\ (http://creativecommons.org/licenses/by-nc/4.0) which \\ permits unrestricted non-commercial use, distribution, and \\ reproduction in any medium, provided the original work is \\ properly cited.
}

pISSN 2093-9043 eISSN 2233-6273 
assess mental health problems in healthcare workers treating COVID-19 patients reported symptoms of depression, anxiety, insomnia, and distress. ${ }^{2}$

For safe and efficient treatment of patients in the COVID-19 era, we describe the current status of neurointervention in Korea affected by COVID-19 based on a nationwide survey and review the literature from other countries and professional societies of neurointervention.

\section{NATIONWIDE SURVEY}

An online survey of Korean Society of Interventional Neuroradiology (KSIN) members was conducted from 14 September 2020 to 14 October 2020 using Google Forms. A total of 62 members (including 31 institutions) participated in the survey. Seventy-three percent of respondents were working at a tertiary medical institution, and $27 \%$ of respondents were working at a secondary medical institution. Topics covered included COVID-19 screening, healthcare worker precaution, neurointervention practice, clinical trial, and mental health (Supplementary Material).

\section{Recommdation for Mechanical Thrombectomy during COVID-19}

To ensure safety from exposure and minimize the treatment delay of large vessel occlusion (LVO)-stroke patients, several professional societies have provided recommendations for decision-making regarding specific steps of management in patients requiring mechanical thrombectomy (MT) during the COVID-19 pandemic., ${ }^{1,3-7}$ Table 1 summarizes the recommendations of the latest publications for MT during the COVID-19 pandemic.

\section{Screening of COVID-19}

\section{Review}

Screening for fever and respiratory symptoms should be part of the screening of all potential neurointervention patients. RT-PCR using a nasal and pharyngeal swab is the current diagnostic gold standard for confirming infection with COVID-19. However, since this test takes 4 to 24 hours depending on the institution, many stroke patients tend to receive a rapid antigen test and chest $C T$ for emergency neurointervention, which can give results within minutes to tens of minutes. The rapid antigen test is relatively cheaper than the RT-PCR test (5.7 vs. 10.8 USD) ${ }^{8}$, and the sensitivity is reported to be $60.5-72.1 \%$, and the specificity is $98.7-99.5 \%$. ${ }^{8,9}$

\section{Survey results}

At the time of the survey, more than half of respondents (54.8\%, 34 of 62 ) conducted an RT-PCR test in patients for emergent neurointervention, and $45.2 \%$ (28 of 62) were selectively performed in patients with suspicious COVID-19 symptoms. The time duration of COVID-19 test results for emergency procedures required $2-24$ hours in $85.5 \%$ of respondents (53 of 62), 1-2 hours in 8.1\% (5 of 62), less than 1 hour in 1.6\% (1 of 62), and more than 24 hours in 4.8\% (3 of 62). Currently, it is mandatory for all patients to have an RT$\mathrm{PCR}$ test in most institutions, and rapid tests can be selectively performed.

- Although the results of RT-PCR for a patient with suspected COVID-19 are not received until the end of the neurointervention procedure, $40.3 \%$ of respondents ( 25 of 62) immediately performed other tasks without waiting for a prophylactic quarantine, and $41.9 \%$ (26 of 62 ) of respondents stopped their work with a temporal quarantine until the test result was available.

- Only 1.6\% (1 of 62) of physicians reported having an experience of treating a COVID-19 positive patient. There was no evidence of COVID-19 infection in the medical staff associated with the neurointervention service.

\section{Chest X-ray and CT}

\section{Review}

Initial chest radiography demonstrated $69-73 \%$ sensitivity in COVID-19 positive patients, but the sensitivity may decrease below 55\% within 2 days of symptoms. ${ }^{10,11}$ Chest CT has substantially improved diagnostic performance over chest radiographs for COVID-19. Typical CT findings of COVID-19 pneumonia include (1) peripheral, bilateral, ground-glass opacities (GGO) (with or without consolidation or visible intralobular lines), (2) multifocal GGOs of rounded morphology (with or without consolidation or visible intralobular lines), and (3) reverse halo signs or other findings of organizing pneumonia (seen in a later stage of disease). ${ }^{12}$ The chest CT sensitivity for COVID-19 relative to RT-PCR was $97-100 \%$. $^{13,14}$ Usually, it is not a suitable screening modality because the proportion of confirmed COVID-19 patients with normal chest CT was relatively high (30.8\%). ${ }^{15}$ However, in emergency neurointervention (including mechanical thrombectomy 
for acute ischemic stroke), chest $\mathrm{CT}$ could be a practical and rapid additional tool (mean acquisition time, $184 \pm 65.5$ seconds; mean radiation dose, $2.47 \pm 1.03 \mathrm{mSv}$ ) for COVID-19 risk stratification prior to RT-PCR results. ${ }^{13}$

\section{Survey results}

In our survey, most respondents $(83.9 \%, 52$ of 62) underwent chest radiography regardless of COVID-19 symptoms when emergency neurointervention procedures were performed. Only $11.3 \%$ of respondents (7 of 62 ) underwent chest CT simultaneously with brain $\mathrm{CT}$ in all patients regardless of COVID-19 symptoms, and $74.2 \%$ of respondents (46 of 62) underwent chest CT simultaneously when COVID-19 symptoms were suspected.

\section{Limiting the Risk of Exposure from Patients}

\section{Review}

Patients with suspected or confirmed COVID-19 must always wear a mask. It is recommended to use a dedicated route (including elevators) from the emergency department to the angiography suite for patients with suspected or confirmed COVID-19. For patients with suspected or confirmed COVID-19 who require intubation before mechanical thrombectomy, it is recommended to intubate them before going to the angiography suite. Performing intubation, extubation, and suction within the angiography suite may increase the risk of viral spread.'

\section{Table 1. Summary of recommendations}

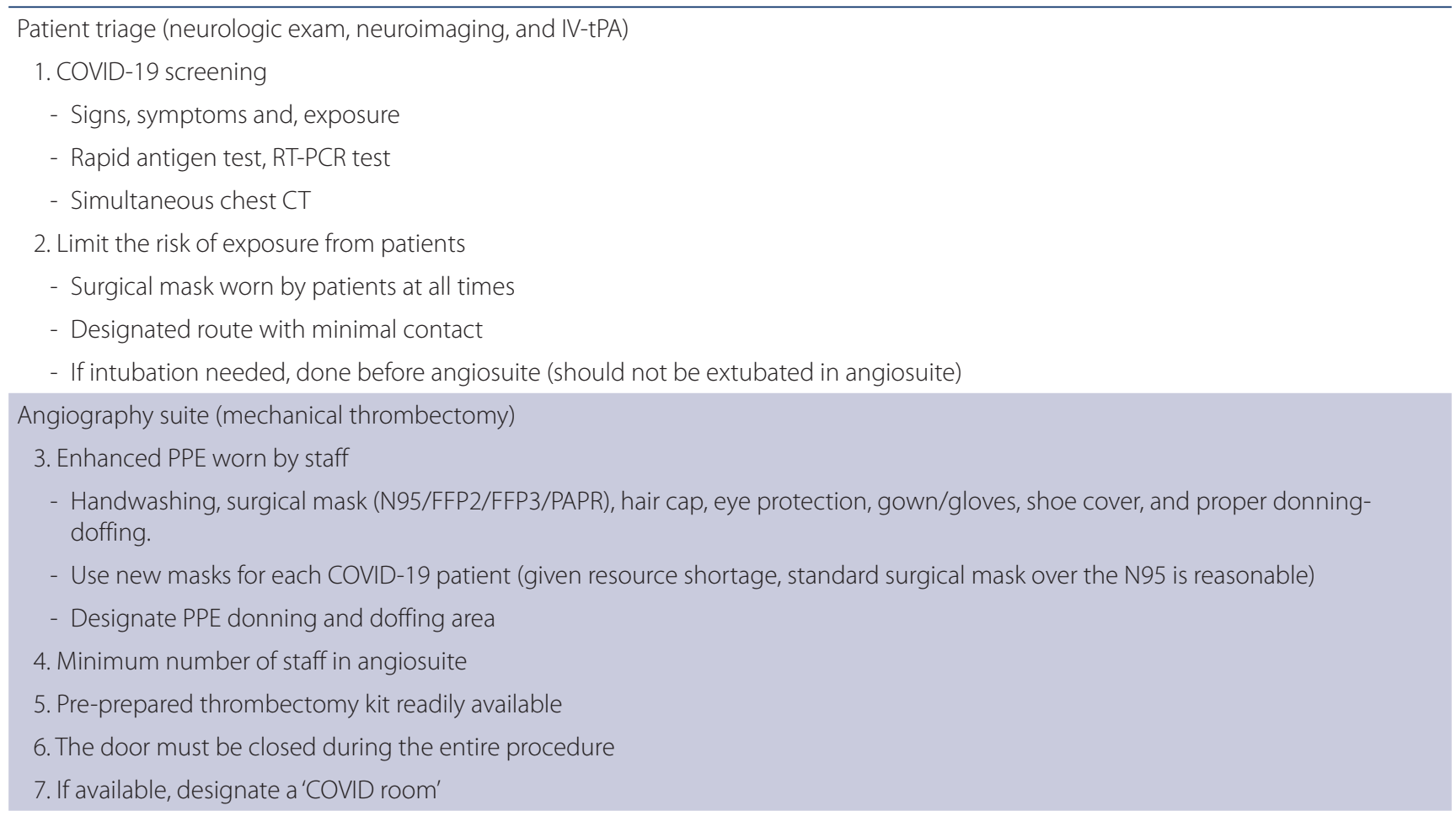

Post-procedural

8. Cleaning angiosuite and equipment

- Exposed surfaces must be cleaned with 70\% ethanol or chlorhexidine-ethanol wipes

- Floors must be cleaned with disinfectant (1:40 diluted bleach, sodium hypochlorite 1/1,000 ppm)

- Immediately after, the angiosuite needs to be ventilated (for 30-60 min) and then wait another 30 min with the door closed before the next patient enters

- If available, negative-pressure ventilation and integrated HEPA filter in angiosuite

IV-tPA, intravenous tissue plasminogen activator; RT-PCR, real-time reverse transcription polymerase chain reaction; $C T$, computed tomography; PPE, personal protective equipment; FFP, filtering face-piece; PAPR, powered air purifying respirator; HEPA, high-efficiency particulate air. 


\section{Survey results}

In our survey, most respondents $(96.8 \%, 60$ of 62$)$ wore a mask if they had suspected or confirmed COVID-19 patients during the neurointervention procedure (without general anesthesia), but 3.2\% (2 of 62 ) did not. Most respondents (82.3\%. 51 of 62) used a dedicated route from the emergency department to the angiography suite if they had suspected or confirmed COVID-19 patients. Intubation for emergency procedures (required for general anesthesia) in suspected or confirmed COVID-19 patients was performed (1) in the angiography suite $(49.2 \%, 30$ of 61$)$, (2) in a separate room with a negative pressure system (24.6\%, 15 of 61$)$, or (3) in a separate room without a negative pressure system (26.2\%, 16 of 61$)$.

\section{Angiography Suite}

\section{Review}

All staff in the angiography suite must use adequate personal protective equipment (PPE) including a surgical mask (N95/ Filtering Face-Piece [FFP]2/FFP3/Powered Air Purifying Respirator [PAPR]), hair cap, eye protection, gown/gloves, and shoe covers, with proper donning-doffing at designated sites. A single-use policy is recommended, but multiple dental masks overlapped on an N95 may be reasonable (given the shortage of equipment). ${ }^{4}$ Limit the number of medical staff within the angiography suite to minimize the exposure from patients. If possible, the neurointervention team should be divided into 2 groups or rotated. ${ }^{3,5}$ Before patient arrival, the preparation of all equipment (e.g., thrombectomy kit) should be completed. Only selected essential equipment should be brought into the angiography suite. Doors must be closed during the procedure.

\section{Survey results}

In our survey, most respondents used a surgical or dental mask $(88.7 \%, 55$ of 62 ) during the neurointervention procedure for suspected or confirmed COVID-19 patients. N95 masks $(6.5 \%, 4$ of 62$)$ and PAPR $(4.8 \%, 3$ of 62$)$ were rarely used. More than half of respondents $(59.7 \%, 37$ of 62$)$ used only 1 mask per day, $25.8 \%$ (16 of 62 ) used 2 masks, 9.7\% (6 of 62 ) used 3 masks, and only $4.8 \%$ (3 of 62) used more than 4 masks. More than half of respondents $(61.3 \%, 38$ of 62) reported wearing PPE adequately during the neurointervention procedure for suspected or confirmed COVID-19 patients and $38.7 \%$ (24 of 62) reported their wear to be insufficient.
- In addition, $27.4 \%$ of respondents (17 of 62) reported having 2 or more teams (fixed divided teams or available to divide depending on the situation) to replace medical staff undergoing quarantine because of COVID-19 exposure. Most respondents $(72.6 \%, 45$ of 62 ) could not be replaced.

\section{Cleaning and Ventilation of the Angiography Suite}

\section{Review}

Access to the current status of an angiography suite ventilation system (positive, negative, filtered, or recirculating air) may assist in the development of a specific workflow to limit viral spread. Isolation of COVID-19 in an angiography suite may not require an extra-ventilation system. However, a dedicated high-efficiency particulate air filtration system may be the best method, and a dedicated negative-pressure system (rather than positive-pressure) with air filtration may be ideal for protecting physicians. ${ }^{3-5}$ After the procedure, cleaning and ventilation of the angiography suite must be ensured. All exposed surfaces within the angiography suite must be cleaned, ventilated for at least 30 minutes, and then it is recommended to wait for 30 minutes with the door closed before the next patient enters. ${ }^{5,16}$ Designating a COVID-19 room is necessary to protect other patients. ${ }^{4}$

\section{Survey results}

Only $16.1 \%$ of respondents ( 5 of 62 ) had an available dedicated negative-pressure system in the angiography suite and most (83.9\%, 57 of 62) did not. Most respondents (93.5\%, 58 of 62) performed cleaning and ventilation of the angiography suite with the door closed after the treatment of a suspected or confirmed COVID-19 patient. Time of ventilation and disinfection after treatment of a suspected or confirmed COVID-19 patient was (1) more than 1 hour 30 minutes (47.5\%), (2) 1 hour (23\%), (3) 30 minutes (23\%), or (4) not performed (6.6\%).

\section{Impact on Mechanical Thrombectomy}

\section{Review}

MT for LVO stroke is highly time-dependent, and rapid initiation of treatment is crucial. Several studies observed a reduction in the number of MT procedures (28-48\%), ${ }^{17-22}$ as well as delayed hospital presentation (last-known well time, increased by 54-160 minutes) $)^{19,20,22}$ and delayed door-to- 
groin puncture time ${ }^{18,20,23,24}$ during the COVID-19 pandemic. Two studies ${ }^{20,24}$ demonstrated no significant difference in the rate of successful reperfusion during the pandemic, and another 2 studies ${ }^{25,26}$ observed increased mortality despite successful reperfusion.

\section{Survey results}

In our survey, $30.5 \%$ (18 of 59, ischemic stroke) and 26.7\% (16 of 60 , hemorrhagic stroke) of respondents reported a reduction (>25\%) in emergency procedures during the pandemic compared with pre-pandemic levels. Only 5.1\% (3 of 59, ischemic stroke) and 5.0\% (3 of 60, hemorrhagic stroke) of respondents indicated an increase in emergency procedures.

- General anesthesia for mechanical thrombectomy was not performed $(93.5 \%, 58$ of 62 ) or rarely performed (3.2\%, 2 of 62) in most respondents before the COVID-19 pandemic, and the practice of general anesthesia for mechanical thrombectomy was not changed after the COVID-19 pandemic in all respondents.

- Procedural details and outcomes of mechanical thrombectomy (delayed presentation, in-hospital delay, reperfusion rate, patient outcome) were not a component of the current survey.

\section{Impact on Clinical Trials}

\section{Review}

Clinical trials of neurointervention have been disrupted following COVID-19 according to the survey by Rai et al. ${ }^{27}$ (65 institutions included). They reported most respondents (78\%) had suspended enrollment for clinical trials (32\% completely or $46 \%$ partially) due to missed trial-related clinics (42\%), missed imaging follow-up (37\%), and protocol deviation (42\%). New clinical trials were also halted in most sites (75\%). Remote consent was available for some studies in $52 \%$ of sites and for all studies in $8 \%$ of sites. They suggested increased use of virtual consent and platform trials may increase enrollment during the pandemic.

\section{Survey results}

In our survey, $42.6 \%$ of respondents (26 of 61) were in ongoing prospective trials including (1) stroke, randomized trial (45.8\%), (2) stroke, single-arm study (45.8\%), (3) aneurysm, randomized trial (0\%), and (4) aneurysm, single-arm study (12.5\%). Among these, $26.9 \%$ of respondents (7 of 26) had an obstacle to continuing in prospective trials due to (multiple responses) delay or disruption in patient recruitment (100\%, 7 of 7), patient follow-up (14.3\%, 1 of 7), or study deviation (14.3\%, 1 of 7). Despite challenges in patient recruitment, no study was discontinued because of patient recruitment. However, some studies had a partial delay $(55.6 \%, 5$ of 9) or were ongoing without delay $(44.4 \%, 4$ of 9$)$.

\section{Impact on Mental Health}

\section{Review}

The physical and mental stress of healthcare workers during the COVID-19 pandemic may be substantial. A recent study to assess mental health problems in healthcare workers treating COVID-19 patients in China (1,257 individuals) reported symptoms of depression (50.4\%), anxiety (44.6\%), insomnia (34.0\%), and distress (71.5\%). ${ }^{2}$

\section{Survey results}

In our survey, half of physicians (48.4\%, 30 of 62) revealed mental and psychological problems including (multiple responses) depression (32.3\%), anxiety (27.4\%), and insomnia (3.2\%).

\section{CONCLUSION}

We found a variety of workflow in various institutions during the COVID-19 pandemic. Full adherence to guidelines against COVID-19 may be challenging. However, specific steps of workflow with individualization according to specific patient conditions and institution circumstances could minimize the risk of COVID-19 spread during neurointervention.

\section{SUPPLEMENTARY MATERIALS}

Supplementary materials related to this article can be found online at https://doi.org/10.5469/neuroint.2021.00304.

\section{Fund}

This study was supported by the Korean Society of Interventional Neuroradiology (KSIN) research grant 2020.

\section{Ethics Statement}

Ethical approval for this study was obtained from the ChungAng University Hospital Institutional Review Board. This 
survey study did not involve patients, and all medical staff members of the survey agreed to participate in the study.

\section{Conflicts of Interest}

The authors have no conflicts to disclose.

\section{Author Contributions}

Concept and design: JSB. Analysis and interpretation: WJL and JSB. Data collection: WJL and JSB. Writing the article: WJL and JSB. Critical revision of the article: WJL. Final approval of the article: JSB. Obtained funding: JSB. Overall responsibility: JSB.

\section{ORCID}

Woong Jae Lee: https://orcid.org/0000-0003-1666-8876

Jun Soo Byun: https://orcid.org/0000-0003-3210-9505

\section{REFERENCES}

1. Smith MS, Bonomo J, Knight WA 4th, Prestigiacomo CJ, Richards CT, Ramser E, et al. Endovascular therapy for patients with acute ischemic stroke during the COVID-19 pandemic: a proposed algorithm. Stroke 2020;51:1902-1909

2. Lai J, Ma S, Wang Y, Cai Z, Hu J, Wei N, et al. Factors associated with mental health outcomes among health care workers exposed to coronavirus disease 2019. JAMA Netw Open 2020;3:e203976

3. Aggour M, White P, Kulcsar Z, Fiehler J, Brouwer P. European Society of Minimally Invasive Neurological Therapy (ESMINT) recommendations for optimal interventional neurovascular management in the COVID-19 era. J Neurointerv Surg 2020;12:542544

4. Fraser JF, Arthur AS, Chen M, Levitt M, Mocco J, Albuquerque FC, et al. Society of Neurolnterventional Surgery recommendations for the care of emergent neurointerventional patients in the setting of COVID-19. J Neurointerv Surg 2020;12:539-541

5. lerardi AM, Wood BJ, Gaudino C, Angileri SA, Jones EC, Hausegger K, et al. How to handle a COVID-19 patient in the angiographic suite. Cardiovasc Intervent Radiol 2020;43:820-826

6. Ospel JM, Goyal M. Endovascular stroke treatment during the COVID-19 pandemic. Nat Rev Neuro/ 2020;16:351-352

7. AHA/ASA Stroke Council Leadership. Temporary emergency guidance to US Stroke Centers during the coronavirus disease 2019 (COVID-19) pandemic: on behalf of the American Heart Association/American Stroke Association Stroke Council Lead- ership. Stroke 2020;51:1910-1912

8. Jakobsen KK, Jensen JS, Todsen T, Tolsgaard MG, Kirkby N, Lippert $F$, et al. Accuracy and cost description of rapid antigen test compared with reverse transcriptase-polymerase chain reaction for SARS-CoV-2 detection. Dan Med J 2021;68:A03210217

9. Brihn A, Chang J, OYong K, Balter S, Terashita D, Rubin Z, et al. Diagnostic performance of an antigen test with RT-PCR for the detection of SARS-CoV-2 in a hospital setting - Los Angeles County, California, June-August 2020. MMWR Morb Mortal Wkly Rep 2021;70:702-706

10. Stephanie S, Shum T, Cleveland H, Challa SR, Herring A, Jacobson $\mathrm{FL}$, et al. Determinants of chest $\mathrm{X}$-ray sensitivity for COVID19: a multi-institutional study in the United States. Radiol Cardiothorac Imaging 2020;2:e200337

11. Wong HYF, Lam HYS, Fong AH, Leung ST, Chin TW, Lo CSY, et al. Frequency and distribution of chest radiographic findings in patients positive for COVID-19. Radiology 2020;296:E72-E78

12. Simpson S, Kay FU, Abbara S, Bhalla S, Chung JH, Chung M, et al. Radiological Society of North America Expert Consensus Statement on reporting chest CT findings related to COVID-19. Endorsed by the Society of Thoracic Radiology, the American College of Radiology, and RSNA - secondary publication. J Thorac Imaging 2020;35:219-227

13. Dhillon PS, Pointon K, Lenthall R, Nair S, Subramanian G, McConachie $\mathrm{N}$, et al. Regional mechanical thrombectomy imaging protocol in patients presenting with acute ischemic stroke during the COVID-19 pandemic. AJNR Am J Neuroradiol 2020;41:1849-1855

14. Ai T, Yang Z, Hou H, Zhan C, Chen C, Lv W, et al. Correlation of chest CT and RT-PCR testing for coronavirus disease 2019 (COVID-19) in China: a report of 1014 cases. Radiology 2020;296:E32-E40

15. Li K, Fang Y, Li W, Pan C, Qin P, Zhong Y, et al. CT image visual quantitative evaluation and clinical classification of coronavirus disease (COVID-19). Eur Radiol 2020;30:4407-4416

16. He Y, Hong T, Wang M, Jiao L, Ge Y, Haacke EM, et al.; Chinese Federation of Interventional and Therapeutic Neuroradiology (CFITN) and the International Society for Neurovascular Disease (ISNVD). Prevention and control of COVID-19 in neurointerventional surgery: expert consensus from the Chinese Federation of Interventional and Therapeutic Neuroradiology (CFITN) and the International Society for Neurovascular Disease (ISNVD). J Neurointerv Surg 2020;12:658-663

17. Amukotuwa SA, Bammer R, Maingard J. Where have our patients gone? The impact of COVID-19 on stroke imaging and intervention at an Australian stroke center. J Med Imaging Radiat 
Oncol 2020;64:607-614

18. Fargen KM, Leslie-Mazwi TM, Klucznik RP, Wolfe SQ, Brown P, Ansari SA, et al. The professional and personal impact of the coronavirus pandemic on US neurointerventional practices: a nationwide survey. J Neurointerv Surg 2020;12:927-931

19. Hajdu SD, Pittet V, Puccinelli F, Ben Hassen W, Ben Maacha M, Blanc $R$, et al. Acute stroke management during the COVID-19 pandemic: does confinement impact eligibility for endovascular therapy? Stroke 2020;51:2593-2596

20. McConachie D, McConachie N, White P, Crossley R, Izzath W. Mechanical thrombectomy for acute ischaemic stroke during the COVID-19 pandemic: changes to UK practice and lessons learned. Clin Radiol 2020;75:795.e7-795.e13

21. Pop R, Quenardelle V, Hasiu A, Mihoc D, Sellal F, Dugay MH, et al. Impact of the COVID-19 outbreak on acute stroke pathways - insights from the Alsace region in France. Eur J Neurol 2020:27:1783-1787

22. Schirmer $C M$, Ringer AJ, Arthur AS, Binning MJ, Fox WC, James RF, et al.; Endovascular Research Group (ENRG). Delayed presentation of acute ischemic strokes during the COVID-19 crisis. J Neurointerv Surg 2020;12:639-642
23. John S, Kesav P, Mifsud VA, Piechowski-Jozwiak B, Dibu J, Bayrlee $A$, et al. Characteristics of large-vessel occlusion associated with COVID-19 and ischemic stroke. AJNR Am J Neuroradiol 2020;41:2263-2268

24. Yang B, Wang T, Chen J, Chen Y, Wang Y, Gao P, et al. Impact of the COVID-19 pandemic on the process and outcome of thrombectomy for acute ischemic stroke. J Neurointerv Surg 2020;12:664-668

25. Bekelis K, Missios S, Ahmad J, Labropoulos N, Schirmer CM, Calnan DR, et al. Ischemic stroke occurs less frequently in patients with COVID-19: a multicenter cross-sectional study. Stroke 2020;51:3570-3576

26. Pop R, Hasiu A, Bolognini F, Mihoc D, Quenardelle V, Gheoca R, et al. Stroke thrombectomy in patients with COVID-19: initial experience in 13 cases. AJNR Am J Neuroradiol 2020;41:20122016

27. Rai AT, Leslie-Mazwi TM, Fargen KM, Pandey AS, Dabus G, Hassan $A E$, et al. Neuroendovascular clinical trials disruptions due to COVID-19. Potential future challenges and opportunities. J Neurointerv Surg 2020;12:831-835 